\title{
STEREOCONTROL INSIDE CONFINED SPACES: ENANTIOSELECTIVE PHOTOOXIDATION OF ENECARBAMATES INSIDE ZEOLITE SUPERCAGES
}

\author{
J. Sivaguru, ${ }^{\dagger}$ Thomas Poon, ${ }^{\ddagger}$ Roberto Franz, ${ }^{\dagger}$ Steffen Jockusch,${ }^{\dagger}$ Waldemar Adam,${ }^{\S}$ and
} Nicholas J. Turro ${ }^{\dagger} * *$.

\begin{abstract}
Dept. of Chemistry and Dept. of Chemical Engineering, Columbia University, 3000 Broadway, Mail Code 3119, New York, NY 10027; ${ }^{\ddagger}$ Joint Science Dept., W.M. Keck Science Center, 925 N. Mills Ave., Claremont McKenna, Pitzer, and Scripps Colleges, Claremont, CA 91711; ${ }^{\S}$ Dept. of Chemistry, University of Puerto Rico, Rio Piedras, PR 00931 and Institut für Organische Chemie, Universität Würzburg, Am Hubland, D-97074 Würzburg (Germany).
\end{abstract}

\section{Contents}

1. General

(Page: 3)

2. Structural Descriptors for the Enecarbamates.

(Page: 3)

3. Procedures

(Pages: 4-6)

(i) General procedure for synthesis of the E-enecarbamates E-1.

(ii) General procedure for photooxidation of enecarbamates 1 in chloroform-d.

(iii) General procedure for photooxidation of enecarbamates 1 inside methylene blue exchanged zeolite.

(iv) Thermal control for enecarbamates 1 inside methylene blue exchanged zeolite.

4. Figures

(Pages: 7-11)

(i) Figure S1: Respective GC traces (Achiral stationary phase) of the photooxidations of oxazolidinone derived Z-enecarbamate Z-1 inside NaY-MB zeolite Top: $10 \mathrm{~min}$. irradiation; Bottom: $30 \mathrm{~min}$. irradiation. Note: the diastereomers of Z-enecarbamates Z-1 are not resolvable by achiral GC and show up as one peak as shown.

(ii) Figure S2: Time dependent photooxidation of Z-enecarbamate Z-1 inside NaYMB zeolite. Note the disappearance of the products $\mathbf{2}$ and $\mathbf{3}$ at longer irradiation 
time. Note: the diastereomers of Z-enecarbamates $\mathbf{1}$ are not resolvable by achiral GC and show up as one peak as shown.

(iii) Figure S3: Respective GC traces (Achiral stationary phase) of photooxidation of oxazolidinone derived $E$-enecarbamate $E-\mathbf{1}$ inside NaY-MB zeolite (10 min. irradiation). Note: the E-enecarbamates $E \mathbf{- 1}$ are resolvable by achiral GC.

(iv) Figure S4: Thermal control: Respective GC traces (Achiral stationary phase) of Oxazolidinone derived Z-enecarbamate Z-1 (top) $E$-enecarbamate $E$-1 (bottom) inside NaY without irradiation. Note: the diastereomers of Z-enecarbamates Z-1 are not resolvable by achiral GC and show up as one peak as shown (top) where as the E-enecarbamates $E \mathbf{- 1}$ are resolvable by achiral GC.

(v) Figure S5: Control Studies: Photooxidation of 4H-Oxazolidinone derived Zenecarbamate Z-5 inside $\mathrm{NaY}$.

5. Table S-1. Time dependence of enantioselectivity - Phototransformation of Z-4(R)3' $(R / S)$-1 inside NaY-MB.

(Page: 12)

6. Figure S6: GC traces of the methyldesoxybenzoin (MDB) product, obtained in the photooxygenation of the Z-1 (top) and $E-\mathbf{1}$ (bottom) enecarbamate diastereomers within methylene-blue-exchanged LiY zeolite.

(Page: 13)

\section{Additional References}




\section{1) General}

NaY (Zeolyst; CBV 100) was used as purchased. Methylene blue (Aldrich), 2,2,4trimethylpentane (Aldrich), Acetonitrile (Aldrich), Hexane (Aldrich), Methanol (Fisher), Methylene chloride (Fisher), methyl tert-butyl ether (Aldrich), 4,4'-di-tert-butylbiphenyl (Aldrich), Chloroform- $d$ (Cambridge Isotope Labs.) were used as received. Product ratios of $R$ and $S$-MDB were obtained using a Varian 3900 gas chromatograph equipped with a Varian CP-Chirasil-DEX CB column as the chiral stationary phase and compared to known samples of MDB as previously reported ${ }^{\mathrm{S} 1}$. Conversions and product yields were determined by GC using 4,4'-di-tert-butylbiphenyl as internal standard and a Varian 3900 gas chromatograph equipped with a Varian Factor-4 VF-1ms column, or by ${ }^{1} \mathrm{H}$ NMR. The Z-enecarbamates 1 were synthesized as previously described. ${ }^{2}$

\section{2) Structural Descriptors for the Enecarbamates.}

We define the following $E / Z-4 \mathrm{R}(R / S)-3^{\prime} \mathrm{R}^{\prime}(R / S)$ structural descriptor for the chiral enecarbamates $\mathbf{1}$, which replaces all previous designations in our former papers and shall be employed in the future. The $E / Z$ specifies the double-bond configuration, the $4 \mathrm{R}(R / S)$ the configuration of the $\mathrm{R}$ substituent at the $\mathrm{C} 4$ position of the oxazolidine ring, and the $3^{\prime} \mathrm{R}^{\prime}(R / S)$ the configuration of the $\mathrm{R}^{\prime}$ substituent in the alkenyl sidechain. For the specific case in this manuscript, the appropriate descriptors would be $Z / E-4^{\mathrm{i}} \operatorname{Pr}(\mathrm{R} / S)-3^{\prime} \operatorname{Ph}(R / S)$. Since throughout this manuscript the $4 \mathrm{R}$ group is always ${ }^{\mathrm{i}} \mathrm{Pr}$ and the $3^{\prime} \mathrm{R}$ ' group always $\mathrm{Ph}$, for convenience the descriptor may be simplified as $Z / E-4(R / S)-3^{\prime}(R / S)$. The complete descriptors for the individual diastereomers used in this manuscript are $Z-4(R)-3^{\prime}(R / S)-\mathbf{1}$, $Z-4(S)-3^{\prime}(R / S)-\mathbf{1}, E-4(R)-3^{\prime}(R / S)-\mathbf{1}$, and $E-4(S)-3^{\prime}(R / S)-\mathbf{1}$. 


\section{3) Procedures}

(i) General procedure for synthesis of the E-enecarbamates E-1.

Approximately $50 \mathrm{mg}$ of a 50:50 mixture of Z-enecarbamate Z-1 [diastereomeric pair $Z-4(R)-3^{\prime}(R / S)$ or $Z-4(S), 3^{\prime}(R / S)$ ] was dissolved in $20 \mathrm{~mL}$ of $\mathrm{CH}_{2} \mathrm{Cl}_{2}$ in a quartz test tube fitted with a septum, gas delivery needle, and vent needle. Samples were purged for 20 min.. with $\mathrm{N}_{2}$ and irradiated at $254 \mathrm{~nm}$ in a Rayonet photochemical reactor for $1 \mathrm{~h}$ under positive $\mathrm{N}_{2}$ pressure. GC analyses showed that the photostationary state $(Z: E=$ 52:48) is reached after $1 \mathrm{~h}$. The solvent was removed under reduced pressure and the resulting residue was loaded onto a $2 \mathrm{~mm}$ thick PTLC plate (EMD Silica gel 60F) and eluted twice with a 2:1 solution of hexanes/methyl tert-butyl ether (MTBE). The highest fraction was isolated to give recovered starting material. The $2^{\text {nd }}$ fraction was extracted with $\mathrm{CH}_{2} \mathrm{Cl}_{2} / \mathrm{MeOH}$ to give from $18-22 \mathrm{mg}$ of a ca 50:50 mixture enecarbamate $E$-1 (diastereomeric pair $E-4(R)-3^{\prime}(R / S)$ or $E-4(S), 3^{\prime}(R / S)$.

(ii) General procedure for photooxidation of enecarbamates 1 in chloroform-d.

An aliquot of each enecarbamate $Z \mathbf{- 1}$ or $E \mathbf{- 1}$ from a standard solution in $\mathrm{CH}_{2} \mathrm{Cl}_{2}$ was added to an NMR tube. The solvent was removed using a stream of $\mathrm{N}_{2}$ and residual solvent was removed by placing the open NMR tube in a vacuum oven at room temperature for at least 2 h. $1.0 \mathrm{~mL}$ of methylene blue $\left(3.7 \times 10^{-4} \mathrm{M}\right)$ in $\mathrm{CDCl}_{3}$ was added and the NMR tube was sealed with a rubber septum and fitted with a gas delivery needle and a vent needle. Oxygen was purged through the sample for $20 \mathrm{~min}$. prior to and during irradiation. Irradiations were performed with a $300 \mathrm{~W}$ halogen lamp with $<500 \mathrm{~nm}$ cutoff filter. After irradiation, samples were analyzed by a GC equipped with achiral stationary 
phase to obtain the mass balance (based on unreacted enecarbamate and the amount of MDB formed after irradiation) and the conversion (based on unreacted enecarbamate).using 4,4'-di-tert-butylbiphenyl as calibration standard. Samples were then analyzed by GC equipped with a chiral stationary phase for determining the enantioselectivity in the product MDB.

(iii) General procedure for photooxidation of enecarbamates 1 inside methylene blue exchanged zeolite.

$300 \mathrm{mg}$ of zeolite loaded with the dye (prepared as in reference S3) was dried in a vacuum oven at $60^{\circ} \mathrm{C}$ for $8 \mathrm{~h}$. The dried dye-loaded zeolite was added to a test tube containing $12 \mathrm{~mL}$ of 2,2,4-trimethyl pentane (isooctane). The dye-zeolite slurry was purged with nitrogen for $15 \mathrm{~min}$. followed by the addition of known amount of enecarbamate $Z$-1 or $E-\mathbf{1}(2-3 \mathrm{mg})$ dissolved in $0.2 \mathrm{~mL}$ of methylene chloride and $1 \mathrm{~mL}$ isooctane. The dye-zeolite slurry was stirred in an oil bath kept at $70^{\circ} \mathrm{C}$ for $5-6 \mathrm{~h}$. The zeolite slurry was then brought to room temperature and fitted with a gas delivery needle and a vent needle. Oxygen was purged through the sample for $20 \mathrm{~min}$. prior to and during irradiation. Irradiations were performed using a $300 \mathrm{~W}$ halogen lamp with a $<500 \mathrm{~nm}$ cutoff filter with stirring and continuous purging of oxygen for a given time interval. The slurry was then filtered and the filtrate (isooctane supernatant) was checked for the presence of the enecarbamate (enecarbamate was not observed in the supernatant). The zeolite residue was transferred to a test tube and extracted by stirring with acetonitrile in an oil bath at $70^{\circ} \mathrm{C}$ for $8 \mathrm{~h}$. The acetonitrile slurry was filtered and the acetonitrile extract was transferred to a round bottom flask. The zeolite powder was further subjected to 
soxhlet extraction overnight with acetonitrile as solvent to confirm complete extraction. The soxhlet extract was combined with the previous acetonitrile extract and then concentrated. The concentrated extract was analyzed using a GC equipped with an achiral stationary phase to obtain the mass balance (based on the unreacted enecarbamate and the amount of MDB formed after irradiation) and the conversion (based on the unreacted enecarbamate) using 4,4'-di-tert-butylbiphenyl as calibration standard. Samples were then analyzed for MDB enantioselectivity using GC equipped with a chiral stationary phase.

(iv) Thermal control for enecarbamates 1 inside methylene blue exchanged zeolite.

$300 \mathrm{mg}$ of zeolite loaded with the dye (prepared as in reference S3) was dried in a vacuum oven at $60^{\circ} \mathrm{C}$ for $8 \mathrm{~h}$. The dried dye-loaded zeolite was added to a test tube containing $12 \mathrm{~mL}$ of 2,2,4-trimethyl pentane (isooctane). The dye-zeolite slurry was purged with nitrogen for $15 \mathrm{~min}$. followed by the addition of known amount of enecarbamate Z-1 or $E \mathbf{- 1}(2-3 \mathrm{mg})$ dissolved in $0.2 \mathrm{~mL}$ of methylene chloride and $1 \mathrm{~mL}$ isooctane. The dye-zeolite slurry was stirred in an oil bath kept at $70^{\circ} \mathrm{C}$ for $5-6 \mathrm{~h}$ and then filtered. The zeolite slurry was then brought to room temperature and filtered. The filtrate (isooctane supernatant) was checked for the presence of the enecarbamate (enecarbamate was not observed in the supernatant). The enecarbamate was extracted and the mass balance was calculated as described above. 

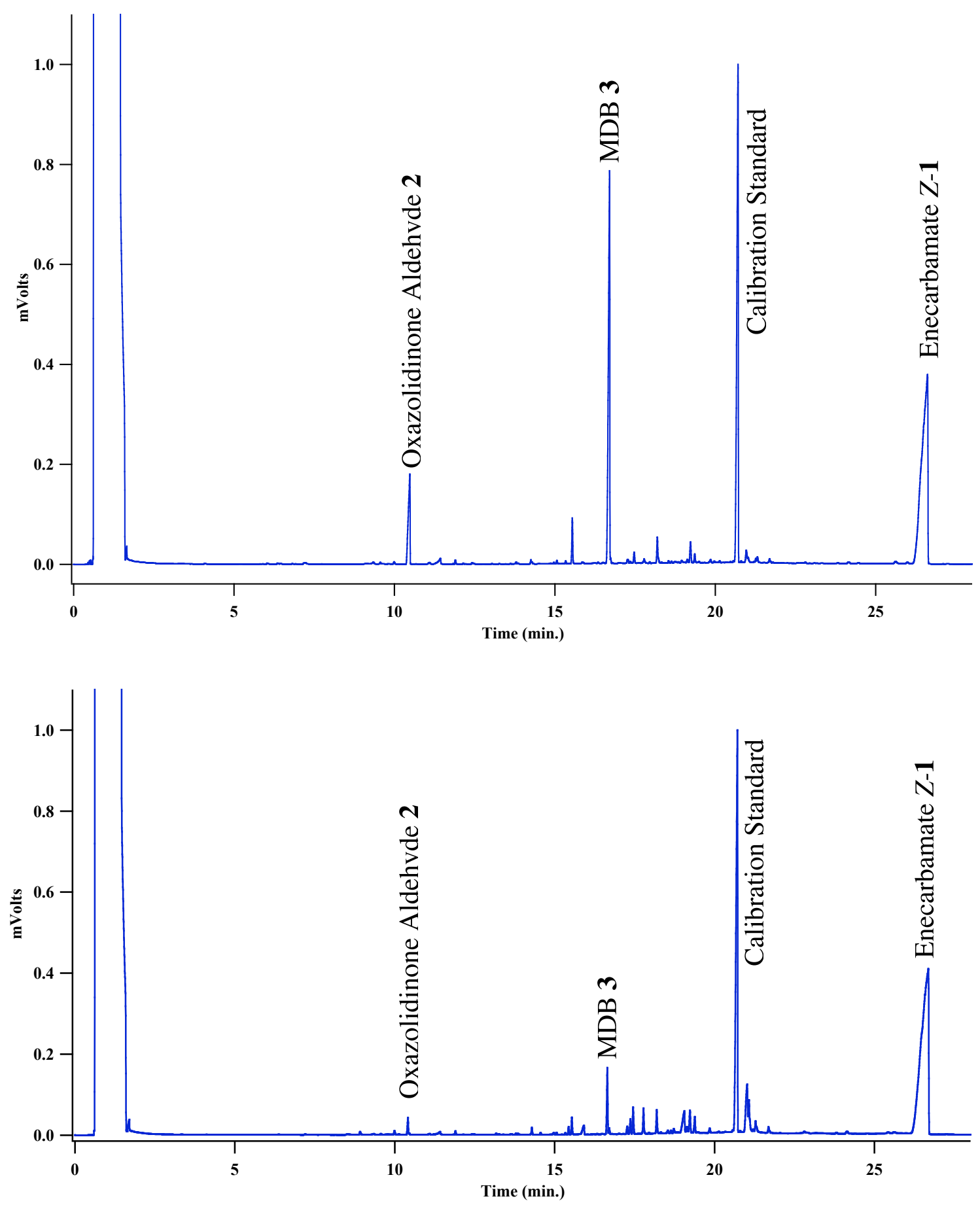

Figure S1: Respective GC traces (Achiral stationary phase) of the photooxidations of oxazolidinone derived $Z$-enecarbamate $Z$-1 inside NaY-MB zeolite Top: 10 min.

irradiation; Bottom: 30 min. irradiation. Note: the diastereomers of Z-enecarbamates Z-1 are not resolvable by achiral GC and show up as one peak as shown. 


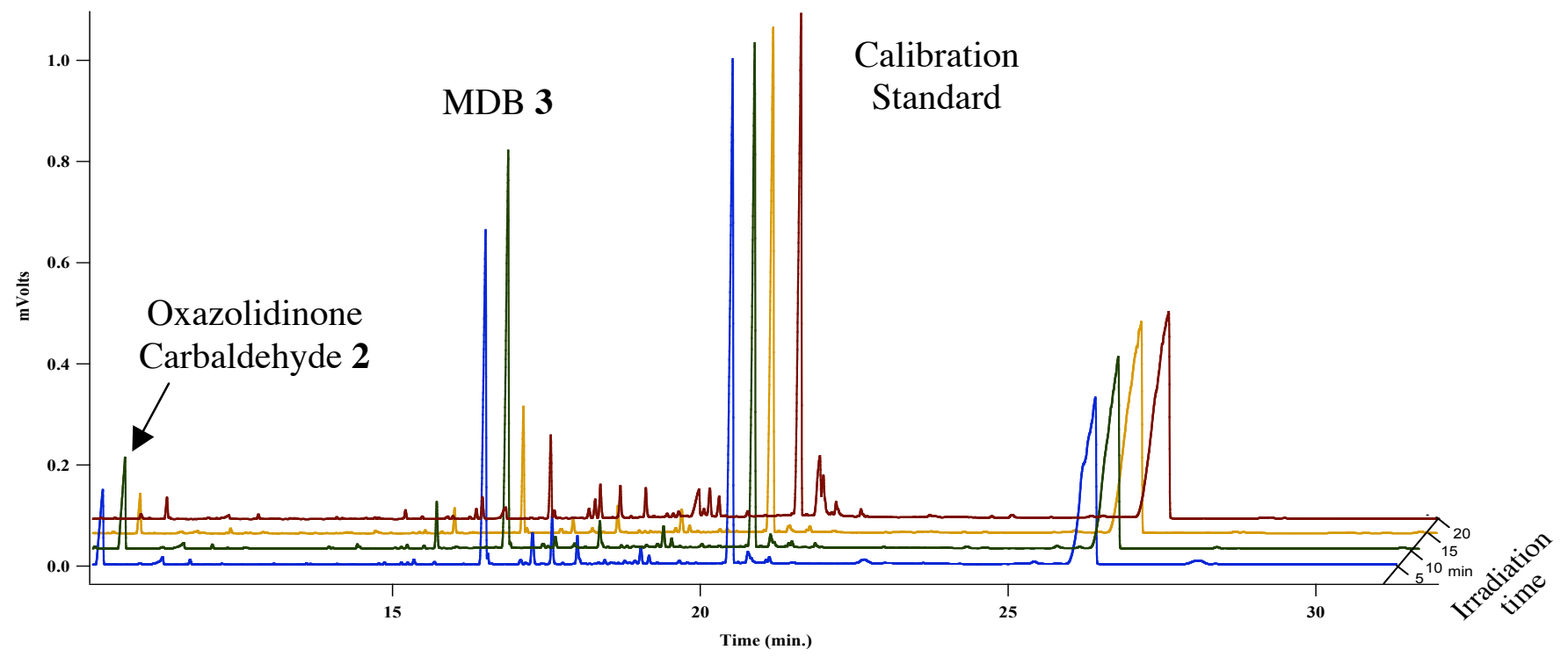

Figure S2: Time dependent photooxidation of $Z$-enecarbamate $Z$-1 inside NaY-MB zeolite. Note the disappearance of the products $\mathbf{2}$ and $\mathbf{3}$ at longer irradiation time. Note: the diastereomers of Z-enecarbamates Z-1 are not resolvable by achiral GC and show up as one peak as shown. 


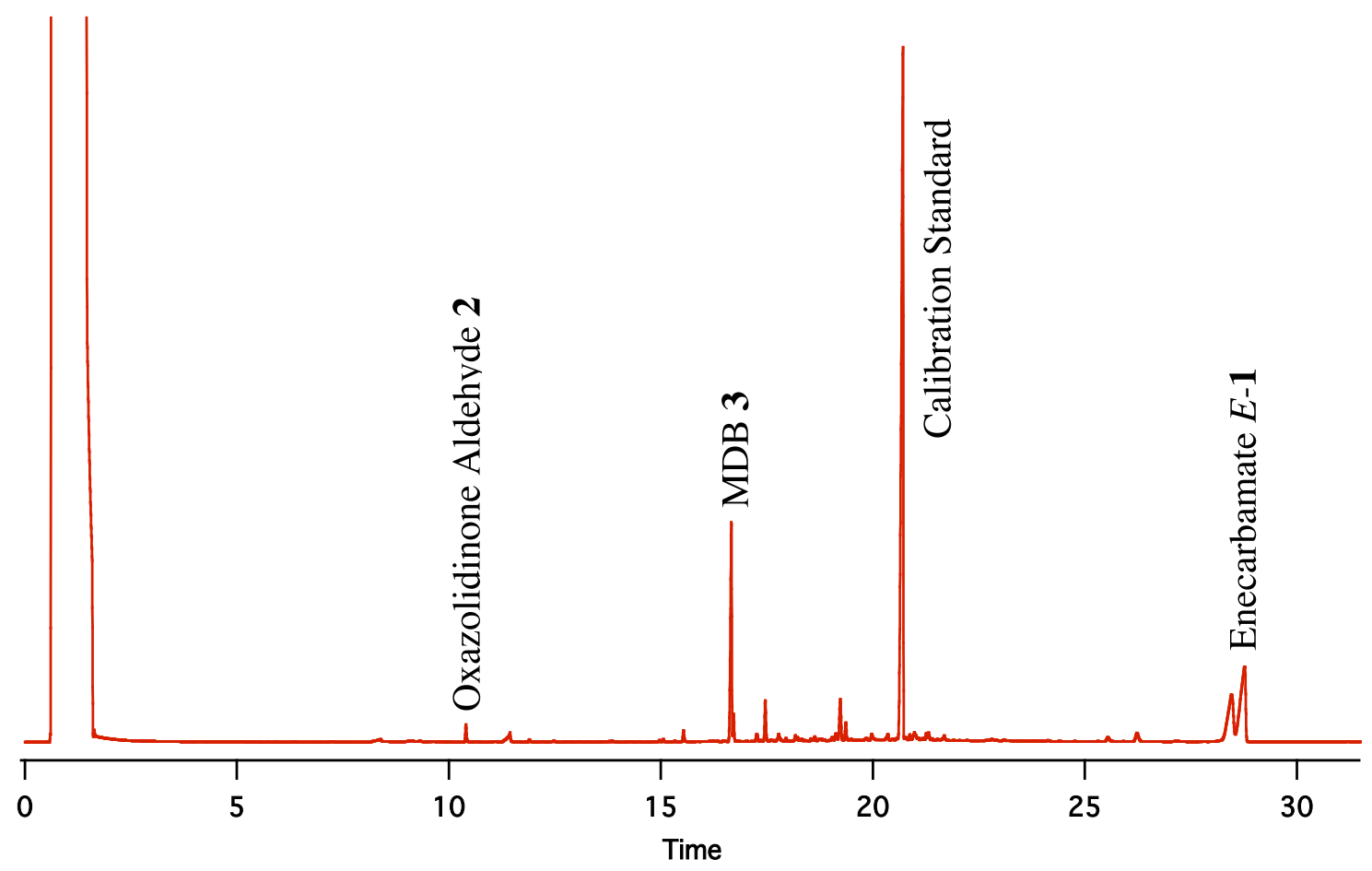

Figure S3: Respective GC traces (Achiral stationary phase) of photooxidation of oxazolidinone derived $E$-enecarbamate $E$-1 inside NaY-MB zeolite (10 min. irradiation). Note: the E-enecarbamates $E-\mathbf{1}$ are resolvable by achiral GC. 

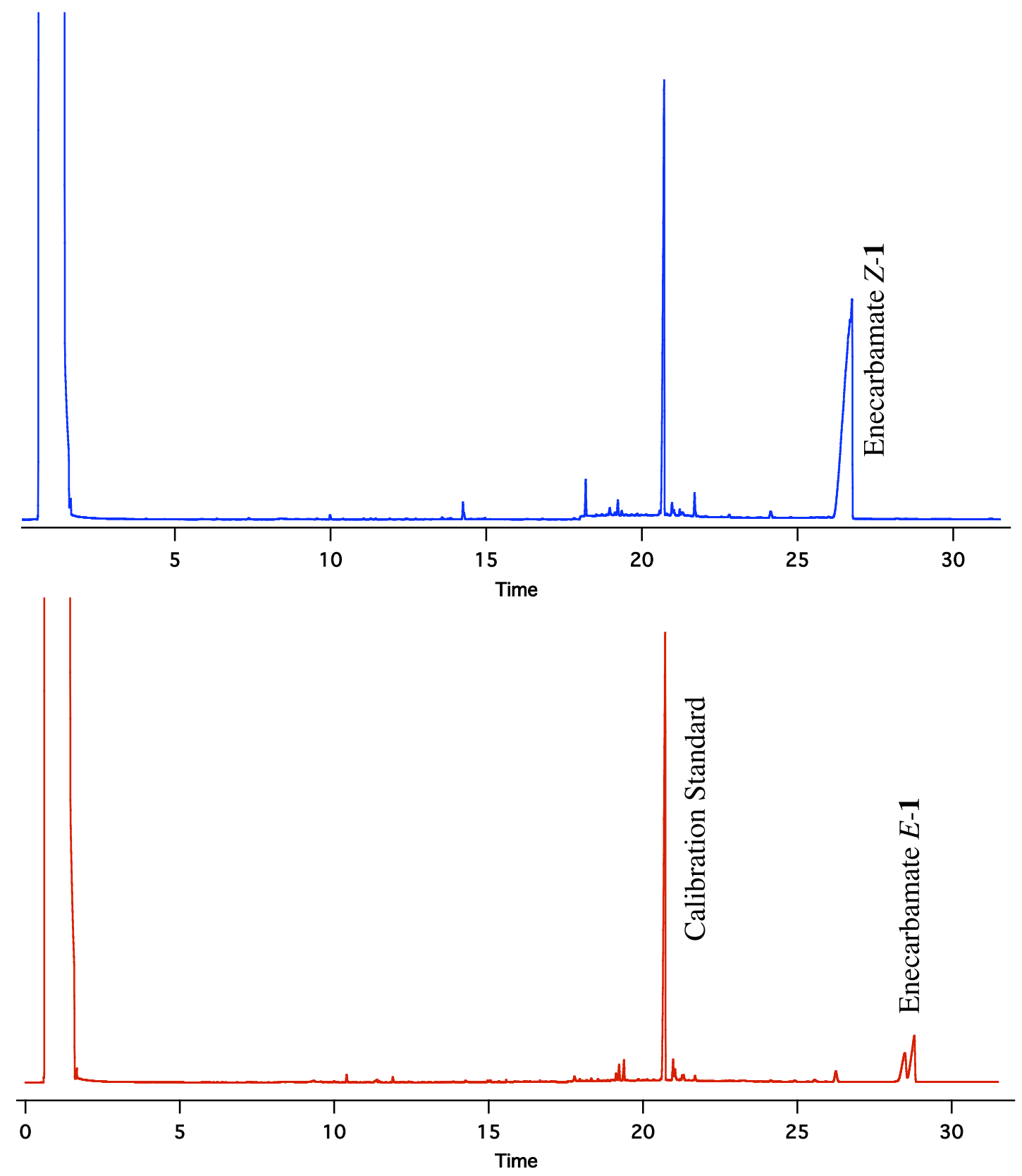

Figure S4: Thermal control: Respective GC traces (Achiral stationary phase) of Oxazolidinone derived $Z$-enecarbamate $Z$-1 (top) $E$-enecarbamate $E$-1 (bottom) inside $\mathrm{NaY}$ without irradiation. Note: the diastereomers of Z-enecarbamates Z-1 are not resolvable by achiral GC and show up as one peak as shown (top) where as the Eenecarbamates $E-\mathbf{1}$ are resolvable by achiral GC. 


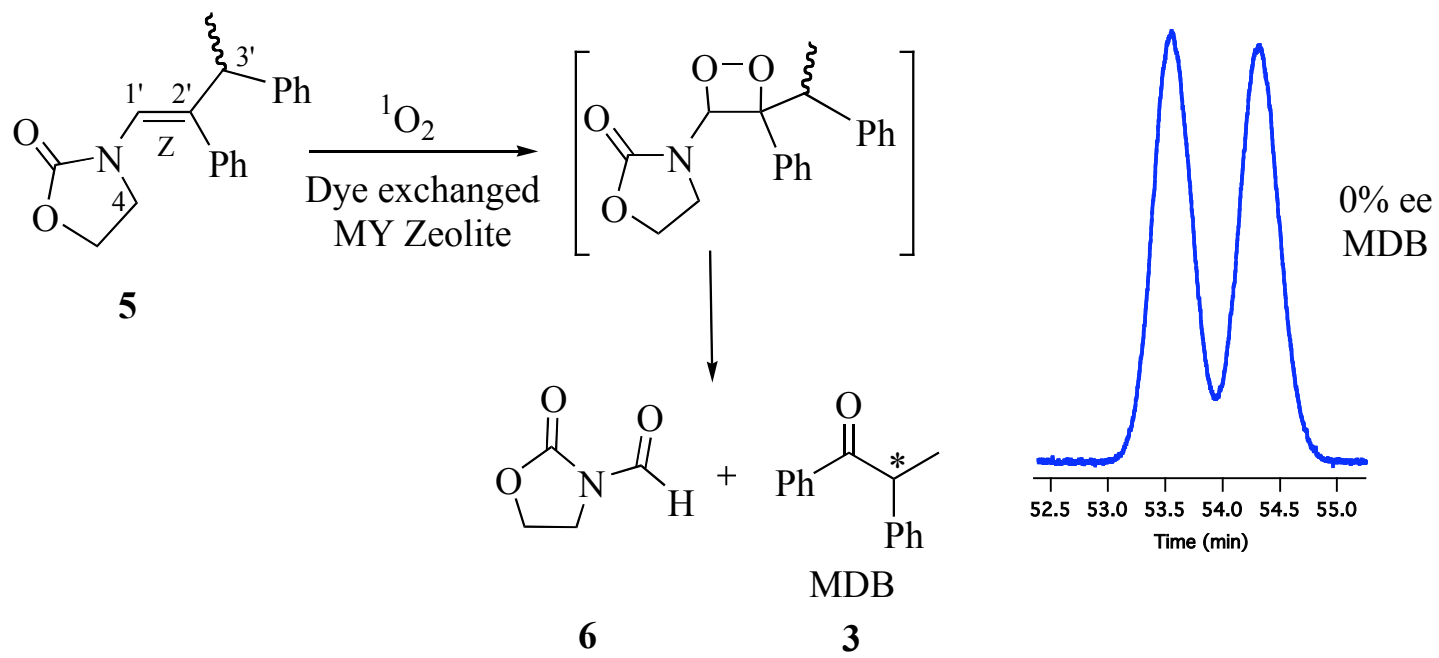

Figure S5: Control Studies: Photooxidation of 4H-Oxazolidinone derived Zenecarbamate Z-5 inside NaY. 
Table S-1. Time Dependence of the Enantioselectivity in the Photooxygenation of the Z-4(R)-3'(R/S)-1 Isomer inside NaY-MB ${ }^{\mathrm{a}}$.

\begin{tabular}{ccc}
\hline $\begin{array}{c}\text { hv time } \\
(\text { min. })\end{array}$ & \%Mass Balance & $\begin{array}{c}\text { \% } \text { ee }^{\mathrm{d}} \\
\text { MDB }\end{array}$ \\
\hline 5 & 79 & $77(\mathrm{~S})$ \\
10 & 86 & $78(\mathrm{~S})$ \\
15 & 65 & $75(\mathrm{~S})$ \\
20 & 55 & $78(\mathrm{~S})$ \\
25 & 50 & $71(\mathrm{~S})$ \\
30 & 58 & $80(\mathrm{~S})$
\end{tabular}

a The methylene-blue loading in NaY was one molecule per 150 supercages (prepared according to the procedure in ref S3), whereas that of the substrate $Z \mathbf{- 1}$ was one molecule per 15 supercages, as a 50:50 3' $(R / S)$ mixture. ${ }^{c}$ Conversion at $5 \mathrm{~min}$. was $38 \%$ and at 10 min. 49\%; the conversions at higher irradiation time were not determined due to the low mass balance; for analytical details, see footnote $\mathrm{c}$ in Table $1 .{ }^{\mathrm{d}}$ Analyzed as in footnote e, Table 1. 

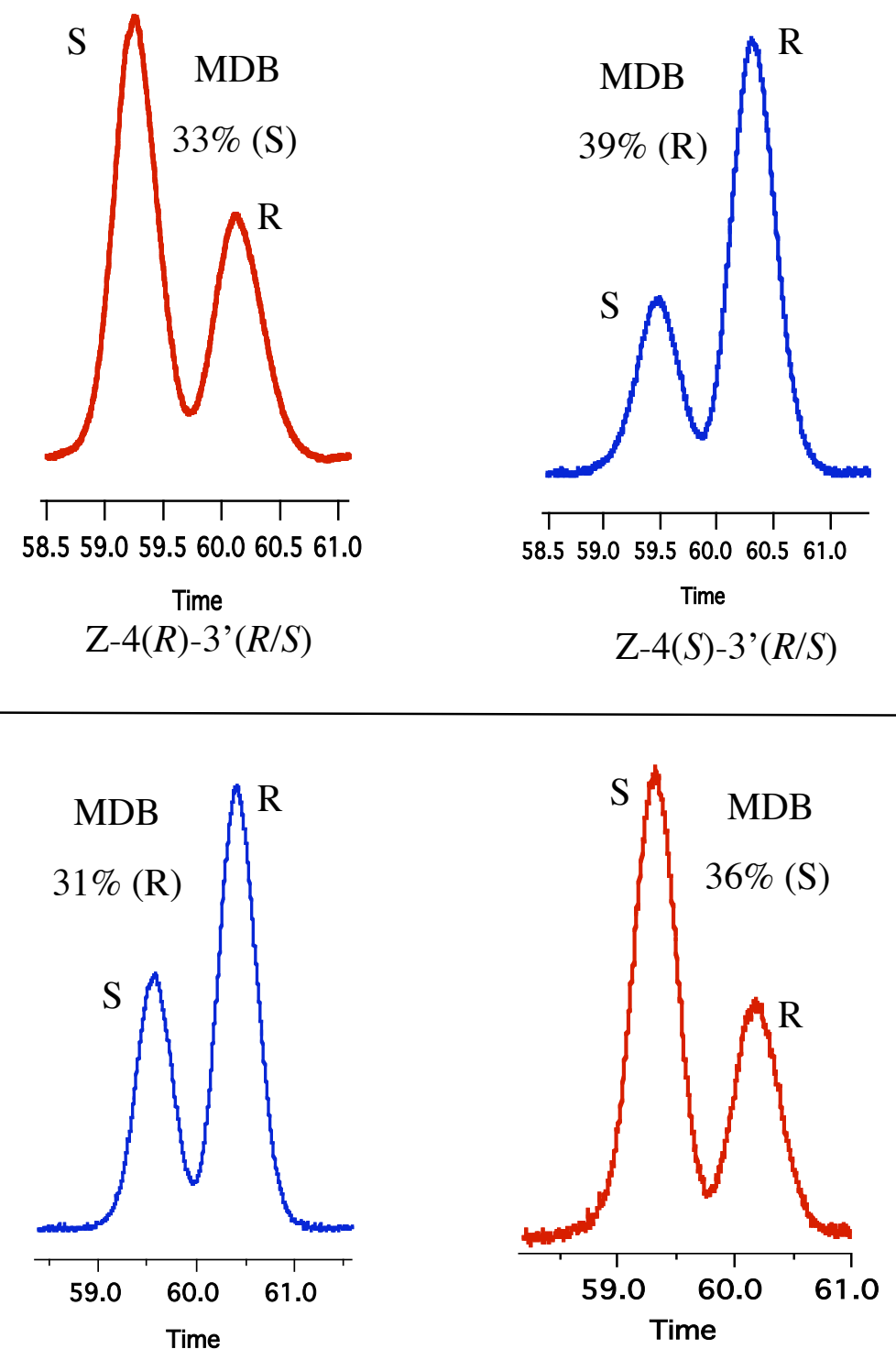

$E-4(R)-3^{\prime}(R / S)$

$E-4(S)-3^{\prime}(R / S)$

Figure S6: GC traces of the methyldesoxybenzoin (MDB) product, obtained in the photooxygenation of the Z-1 (top) and E-1 (bottom) enecarbamate diastereomers within methylene-blue-exchanged LiY zeolite. 


\section{Additional References}

S1 Poon, T.; Turro, N. J.; Chapman, J.; Lakshminarasimhan, P.; Lei, X.; Adam, W.; Bosio, S. G. Org. Lett. 2003, 5, 2025-2028.

S2 Procedures and product characterizations for the $Z$-enecarbamates can be found in the first paper in this series: Adam, W.; Bosio, S. G.; Turro, N. J.; J. Am. Chem. Soc. 2002, 124, 8814-8815.

S3 Shailaja, J.; Sivaguru, J.; Robbins, R. J.; Ramamurthy, V.; Sunoj, R. B.; Chandrasekhar, J. Tetrahedron, 2000, 56, 6927-6943. 